\title{
Development and Research of Root File System Based on Cortex-A8 Microprocessor
}

\author{
Li Zhou ${ }^{1}$ and Maoqun $\mathrm{Yao}^{2, *}$ \\ ${ }^{1}$ Institute of Service Engineering, Hangzhou Normal University, Hangzhou Zhejiang 311121, China \\ ${ }^{2}$ Institute of Service Engineering, Hangzhou Normal University, Hangzhou Zhejiang 311121, China \\ *Corresponding author
}

\begin{abstract}
The root file system is an integral part of the embedded Linux system. Busybox is used in the embedded environment to build a root file system, the paper mainly studies how to complete the root file system based on Busybox. This article by root in the ubuntu configuration, compile Busybox and build the root file system necessary files to make the root file system. Finally, successfully mount the experiment by nfs remote mount the root file system in the host ubuntu. The method described in the article can be successfully run in the ARM development board, to provide the necessary environment for the development of embedded systems.
\end{abstract} $n f s$

Keywords-Linux system; root file system; Busybox; Ubuntu;

\section{INTRODUCTION}

In the Linux system, the file system is a set of software whose function is to manage the sectors of the storage device. When the user accesses a file according to the file name at the application layer, the file system converts the file name to access to the sector number, thereby realizing that all devices in the Linux system are files. The root file system is a file system first. It not only has the function of storing data files, but also the first file system mounted at kernel startup. The kernel code image file is stored in the root file system, and the system boots the startup program. After loading the root file system, some initialization scripts and services are loaded into memory to run. The root file system provides the most basic link libraries, scripts, and special files for Linux system operation. In a complete Linux system, only the kernel itself can not work, like common Linux systems such as ubuntu, redhat, centos, etc. must be in the root file system etc directory under the configuration file / bin, / sbin under the shell directory The commands, as well as the library files in the /lib directory, work together. In other words, the root file system provides the Linux root directory. The common $\mathrm{ls}$, cd, and pwd commands are implemented on the root file system. The root file system also includes the init process, which implements a leap from kernel mode to user mode ${ }^{[1]}$. This article will make a good root file system in ubuntu through NFS remote mount to the s5pv210-based development board, and gives the test results.

\section{ROOT FILE SYSTEM}

\section{A. Root File System Introduction}

The embedded Linux system can be summarized as an application layer and a kernel layer. The application layer is mainly user software. The kernel layer includes a root file system, an operating system, and the underlying hardware. ${ }^{[2]}$ The architecture of its components is shown in Figure 1. The specific directories and functions of the Linux root file system are shown in Table 1.

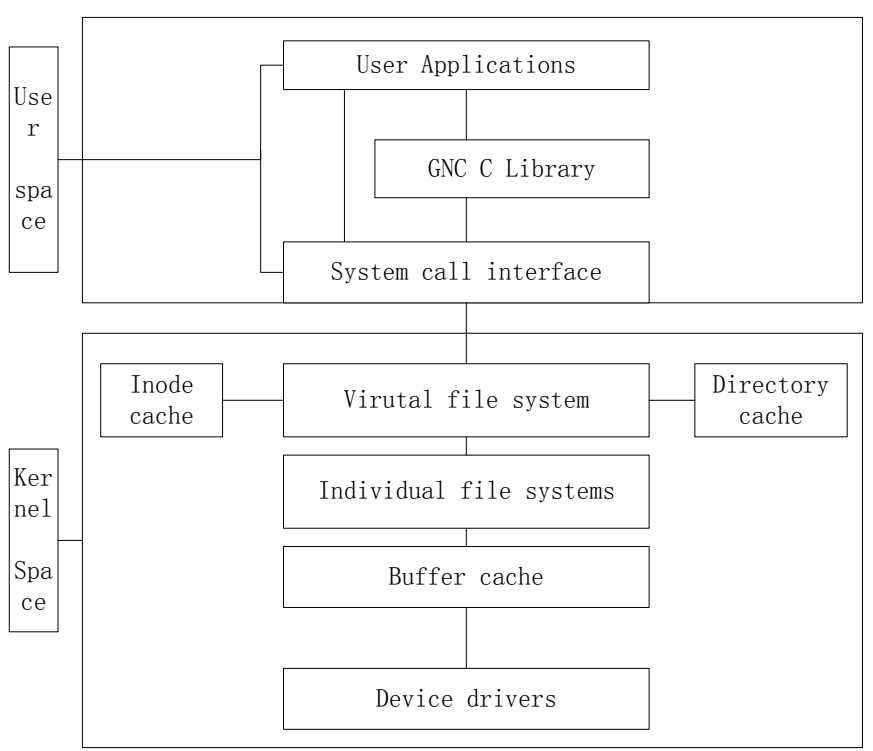

FIGURE I. LINUX SYSTEM ARCHITECTURE

TABLEI. LINUX ROOT FILE SYSTEM DIRECTORY TABLE

\begin{tabular}{|c|c|c|}
\hline \multirow{13}{*}{$\begin{array}{l}\text { /(Root } \\
\text { directory) }\end{array}$} & /bin & Store linux general user command directory \\
\hline & /sbin & Storage administrator system commands \\
\hline & /dev & Storage device file \\
\hline & /etc & Store various configuration files \\
\hline & /lib & Store shared libraries and loadable drivers \\
\hline & /root & Root user \\
\hline & /usr & Store shared, read-only programs and data \\
\hline & $/ \mathrm{mnt}$ & $\begin{array}{l}\text { Provide users with temporary mounting of other } \\
\text { file systems }\end{array}$ \\
\hline & /opt & Additional installation software location \\
\hline & $/ \operatorname{tmp}$ & The directory where temporary files are stored \\
\hline & /var & Store variable data, log files \\
\hline & /media & Storage and mounting equipment \\
\hline & /proc & The mount point of the file system \\
\hline
\end{tabular}




\section{B. Build a Root File System}

Create an empty root file system file in the host ubuntu and name it rootfs. Then create necessary subdirectories such as bin, sbin, dev, etc, lib, var, etc. in the rootfs directory. The specific operations are as follows ${ }^{[3]}$ :

mkdir rootfs

cd rootfs

mkdir bin dev etc proc root sbin sys tmp usr var

\section{THE TRANSPLANT OF BUSYBOX}

Busybox is a big toolbox that integrates many basic commands for Linux systems, such as 1s, cd, echo, and so on. The Busybox configuration can be done in menuconfig just like the kernel configuration. Busybox is also an open source project. All source code can be downloaded from the Internet. In this research, Busybox makes its own root file system.

\section{A. Modify Makefile and CROSS_COMPILE}

Graphics may be full colour but make sure that they are In the official website to download Busybox1.24.1 to the host ubuntu system, decompression is completed, the first step to modify the Makefile, because the development board uses the cpu Samsung s5pv210 belongs to the Cortex-A8 series architecture, so its ARCH belongs to the arm series, In the ubuntu system to find the cross compiler tool chain installation, copy the relevant path to the Makefile CROSS_COMPILE, the specific code is modified as follows ${ }^{[\overline{4}, 5]}$ :

$\mathrm{ARCH}=$ arm

CROSS_COMPILE=/usr/local/arm/arm-2009q3/bin//armnone-linux-gnueabi-

\section{B. Configure Busybox}

Bussybox in the menuconfig related configuration, and then compiled, installed into the rootfs directory.

The Busybox runtime needs to be independent of other libraries. When Busybox is configured, Busybox is compiled into a statically linked executable file. Specific steps are as follows ${ }^{[6]}$ :

Busybox Settings--->

Build Options--->

shared libs)

[*]Build BusyBox as a static binary(no

\# Vi style line editing commands

Busybox Library Tuning--->

[*]vi-style line editing commands

[*]Fancy shell prompts

\# Uncheck Simplified modutils, use full tool command

Linux Module Utilities--->

[ ]Simplified modutils

$[*]$ insmod

[*]rmmod

[*]lsmod

[*]modprobe

[*]depmod

\# Make sure mdev is supported

Linux System Utilities--->[*]mdev

[*]Support /etc/mdev.conf

[*]Support subdirs/symlinks
[*]Support regular expressions substitutions when renaming dev

[*]Support command execution at device addition/removal

[*]Support loading of firmwares

\section{BuILD A RoOT FILE SySTEM}

After Busybox is compiled and installed into the specified rootfs directory, the corresponding tool files are generated in the /bin, /sbin, and /usr directories. The necessary configuration files such as inittab, rcS, etc. must also be added to enable the root file to be created. The system works ${ }^{[7]}$.

\section{A. Build Inittab File}

Inittab is a runtime configuration file in the /etc directory. When the Linux system is running, it parses the text file and decides how to work according to the contents of the resolution, including the user's run level settings, login system settings, restart settings, etc. Its format is defined in Busybox, and it is parsed in line units. The configuration items for each line of the inittab text file are determined by the four configuration values id: runlevels: action: process. The two configuration values, id and runlevels, can be left blank. Action is a conditional state, and process is the path name of a program that can be executed ${ }^{[8]}$. That is, the process will be executed when the conditions of the action are satisfied. The build code looks like this:

\#Run the system script file

::sysinit:/etc/init.d/rcS

s3c2410_serial2::sysinit:/bin/login

::sysinit:/bin/login

::ctrlaltdel:-/sbin/reboot

\# Remove all file systems

::shutdown:/bin/umount -a -r

\# Restart the init process

::restart:/sbin/init

\section{B. Build an rcS File}

The $\mathrm{rcS}$ file is the most important file in the Linux runtime configuration file. Other configurations are derived from the $\mathrm{rcS}$ file, which is located in the /etc/init.d directory. In this file, the PATH environment variable is an environment variable defined inside the Linux system. This ensures that the user can directly use Linux system commands such as 1s, cd, and pwd when entering the command line. Set the system run level in the file to single-user mode, ie runlevel=S. Set the file to userreadable and writable mode, umask $=022$. Use mount-a to mount all filesystems. Of course, all mount points /proc, /sys, /var, /tmp, and /dev must be created in the root directory. The file can also be used to set the host name and host network card ip. The specific operation is shown in the following code $^{[9]}$.

\# Set the environment variable

PATH=/sbin:/bin:/usr/sbin:/usr/bin

\# Set user mode to single user mode

Runlevel $=\mathrm{S}$

Prevlevel $=\mathrm{N}$

\# Set the file permissions to 022 and user default permissions to 644

Umask 022

\# Export environment variables 
Export PATH runlevel prevlevel

\#mount all devices

Mount -a

\# Generate dev directory related content

Echo $/ \mathrm{sbin} / \mathrm{mdev}>/ \mathrm{proc} / \mathrm{sys} /$ kernel/hotplug

Mdev -s

\# Set the host name, here set to zhouli

/bin/hostname -F/etc/sysconfig/HOSTNAME

\# Set the host network card ip

Ifconfig eth0 172.17.64.188

\section{Build a Device Driver File}

\section{1) Build fstab file}

After the mount point is created in the rootfs directory, the mount-a command in the Busybox parsing the rcS file will look for the file /etc/fstab. This file will list all the files that should be mounted in a certain format. System, build code is as follows ${ }^{[9]}$ :

\# File System Mount Point Type Options Backup Verify Sectors

$\begin{array}{llll}\text { proc } & \text { /proc proc } & \text { defaults } 0 & 0 \\ \text { sysfs } & \text { /sys sysfs } & \text { defaults } 0 & 0 \\ \text { tmpfs } & \text { /var tmpfs defaults } & 0 & 0 \\ \text { tmpfs } & \text { /tmp tmpfs defaults } & 0 & 0 \\ \text { tmpfs } & \text { /dev tmps } \text { defaults } & 0 & 0 \\ 2 \text { ) Configure mdev } & \end{array}$

In Linux system, udev is a device manager. Its main function is to manage device nodes in the dev directory. mdev is a simple udev that comes with Busybox. It is an application layer software, under the /dev directory. Device driver files are generated by medv. When the rcS file does not start mdev, there is no device driver file in the /dev directory. Add related mdev-related configuration in the $\mathrm{rcS}$ file, as follows:

\# Generate dev directory related content

Echo /sbin/mdev > /proc/sys/kernel/hotplug

Mdev -s

After restarting the system, use the ls command to view a lot of device driver files generated in the / dev directory, indicating that the device driver file is successfully built, as shown in Figure $2^{[10]}$ :

\begin{tabular}{|l|l|} 
ptȳi 7 \\
ptyq8 \\
ptyq9 \\
ptyqa \\
ptyqu \\
ptyqc \\
ptyqd \\
ptyqe \\
ptyqu \\
ptyro \\
ptyr1 \\
ptyr2 \\
ptyr 3 \\
ptyr4
\end{tabular}
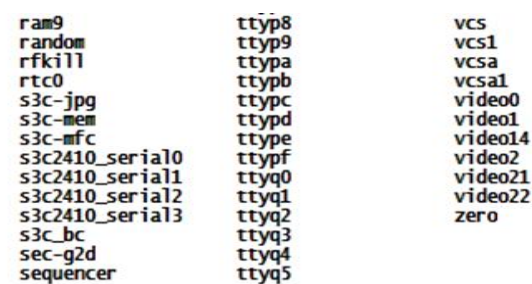

FIGURE II. DEVICE DRIVER FILE MAP

\section{Build an rcS File}

During the Linux system running, the /etc/profile file is automatically invoked by Busybox. The environment variables set in the profile file affect all users in the Linux system $^{[11]}$. A typical profile is built in the /etc directory. The code looks like this:

\# Get the currently logged in user name

USER="'id -un'"

\# The current user's login name
LOGNAME $=\$$ USER

\# basic prompt, \# for root user, \$ for normal user

PS1='[lu@ \h \W] \#'

\# Determines which directories the shell will find commands or programs

PATH $=\$$ PATH

\# Current host name

HOSTNAME=`/bin/hostname`

\# Export environment variables

Export USER LOGNAME PS1 PATH

\section{E. Set up User Login}

\section{1) Login interface settings}

There is a user login interface on Linux systems. In this experiment, a simple character interface was created to interact with the user ${ }^{[11]}$. In the /etc/sysconfig/HOSTNAME file can be used to set the name of the prompt in the development board command line, in the HOSTNAME file to set the prompt name to "zhouli", so that the command line prompt will be displayed in front of [@zhouli] \#. Since the configuration item s3c2410_serial2::sysinit:/bin/login has been set in the inittab file, the login will not enter the command line and directly enter the login interface. The specific implementation command is as follows:

vi /etc/sysconfig/HOSTNAME

zhouli

\section{2) Username and password settings}

The files used to describe user names and passwords on Linux systems are passwd and shadow files, both of which are in the letc directory. The passwd file stores the user's login password, and the shadow file stores the encrypted password. Directly copy the /etc/passwd and /etc/shadow files from the Ubuntu system to the currently created rootfs directory ${ }^{[12]}$. After the corresponding changes are made, the login user name on the development board is root, and the password is the root user of the ubuntu system. password. The specific order is as follows:

$\mathrm{cp} /$ etc/passwd /etc/shadow ./rootfs

\section{NFS MOUNT}

NFS is a network communication protocol consisting of a server and a client. The use of nfs in this production is mainly used as a mount of the root file system. In the host ubuntu system set up a configured nfs server, the kernel running in the development board as a client of nfs. In the host ubuntu's nfs server, export the rootfs folder in the form of a folder. In the development board, you can mount the root file system in this folder and start the kernel on the development board. Starting the kernel in this way is equivalent to the remote mounting of the kernel on the development board to the root file system in the host ubuntu. The specific NFS configuration is as follows:

\section{A. Install nfs}

After installing nfs in ubuntu with the sudo apt-get install nfs-kernel-server and sudo apt-get install nfs-common commands, open /etc/exports and add /root/rootfs *(rw,sync, No_root_squash, no_ subtree_ check), that is, the root file system in /root/rootfs is mounted on the development board. You also need to use chmod 777 -R /root/rootfs to modify the permissions so that the development board can directly 
execute the ubuntu program. Sudo /etc/init.d/nfs-kernel-server restart Restart the nfs service.

\section{B. Nfs Configuration}

\#IP to support kernel configuration

[*] Networking support --->

Networking options --->

[*]IP: kernel level autoconfiguration

\# File system supports nfs client and making root file system with nfs

File systems --->

[*] Network File Systems --->

--- Network File Systems

$<*>$ NFS client support

[*]NFS client support for NFS version 3

[*]NFS client support for the NFSv3 ACL protocol extension

[*]NFS client support for NFS version 4 (EXPERIMENTAL)

[*]NFS client support for NFSv4.1 (DEVELOPER ONLY)

[*]Root file system on NFS

\section{Set Uboot Bootargs}

When uboot starts, it will pass parameters to the kernel via bootargs, set uboot's bootargs such as the path of the root directory, host IP, development board IP, gateway, serial port, baud rate, etc. to support the nfs way to mount the root file in ubuntu. System, the specific settings are as follows:

setenv bootargs $\mathrm{root}=/ \mathrm{dev} / \mathrm{nfs}$

nfsroot=172.17.64.141:/root/porting_x210/rootfs

$\mathrm{ip}=172.17 .64 .188: 172.17 .64 .167: 172.17 .64 .1: 255.255 .255 .0:$ :

eth0:off init=/linuxrc console $=$ ttySAC2,115200

After the setup is complete, start the Linux system on the development board, $\log$ in as the root user, and successfully enter the shell command line. You can use the shell command to prove that the nfs mounts the root file system remotely. Some of the experimental processes are shown in Figure 3. The result is shown in Figure 4:

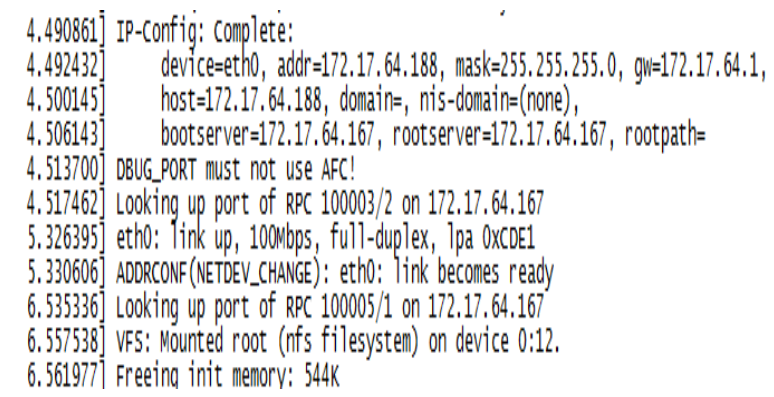

FIGURE III. NFS MOUNT STARTUP PROCESS DIAGRAM

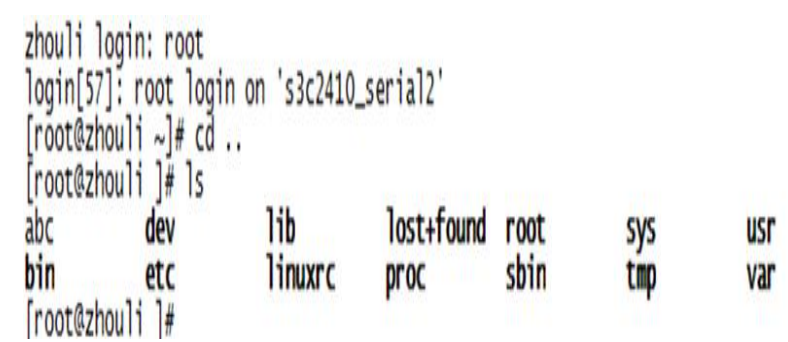

FIGURE IV. SUCCESSFUL MOUNTING PHENOMENON

\section{CONCLUSION}

In an embedded Linux system, a good root file system can maximize resource utilization and improve overall system performance. This article begins with an empty folder, describes in detail the composition, transplantation, production, and mounting of the root file system. It is believed that there will be great help for the study of the embedded Linux file system.

This article innovation: the general root file system is built on the original file to cut, in this article, the use of Busybox plus necessary files to create a new root file system, in this process elaborated inittab, rcS, etc. The composition of important files, when testing the experimental results, uses nfs to mount the root file system remotely. Finally, the root file system that has been successfully created is successfully run on the development board.

\section{ACKNOWLEDGEMENTS}

Current-mode CMOS digital and integrated circuit theory and technology research (61771179), Research on the design of threshold arithmetic algebraic system and current-mode CMOS circuit (LY15F010011)

\section{REFERENCES}

[1] Matt Welsh \& Lar Kaufman. "The Definitive Guide to Linux" [M]. Hong Feng translated. 3rd edition. China Electronic Publishing, 2000.

[2] Barr. Linux File System [M]. Tsinghua University Press, 2003.

[3] Zou Sibiao. Embedded Linux Design and Application [M]. Tsinghua University Press, 2002.

[4] Ma Lijie. Embedded Linux Application Programming [M]. Beijing Institute of Technology Press, 2016.

[5] Yan Haitao, Nie Hongyi, Chen Qingwen. The Creation and Porting of Root File System Based on Embedded Linux[J]. Automation Technology and Applications, 2014, 33(12):21-24.

[6] Peng Hao, Gong Jie, Qin Jianmin. Embedded Linux Root File System Based on S3C2440[J]. Electronic Design Engineering, 2010, 18(6):2022.

[7] Liu Ergang. A Method of Constructing Embedded Linux Root File System [J]. Electronic Design Engineering, 2016, 24(9):160-162.

[8] Xiong Xingxing, He Yueshun. U-boot Analysis and Porting Based on S5PV210[J]. Journal of Computer Systems, 2015, 24(1):199-205.

[9] Du Haixing. Embedded Bootloader Analysis and Porting Based on ARM[J]. Microcomputer Information, 2010, 26(29):58-59+57.

[10] Blum R. Linux Command Line and Shell Scripting Bible[M]. John Wiley \& Sons, 2015.

[11] Nam S, Yoon S K, Kim S D. Fast bootstrapping method for the memory-disk integrated memory system[C]// Ieee/acis, International Conference on Computer and Information Science. IEEE, 2015:167-172.

[12] Wang L. Realization of U-Boot booting through NAND Flash[J]. Electronic Design Engineering, 2010. 